\title{
Avaliação do desempenho da equipe de enfermagem na administração de fármacos via intubação gastrointestinal
}

Nursing team's performance evaluation regarding the administration of drugs via gastrointestinal intubation

\section{Evaluación del desempeño del equipo de enfermería en la administración de fármacos vía intubación gastrointestinal}

Dayana Craveiro Ramos ${ }^{1}$, Joselany Áfio Caetano ${ }^{2}$, Jennara Candido Nascimento ${ }^{3}$, Bertiane Maria Gadelha de Freitas ${ }^{4}$, Liana Mara Rocha Teles 5 , Maira Di Ciero Miranda ${ }^{6}$

\section{RESUMO}

Esta pesquisa objetivou avaliar o desempenho de técnicos e auxiliares de enfermagem durante o preparo e a administração de fármacos via intubação gastrointestinal. Trata-se de estudo prospectivo, observacional, realizado em um hospital universitário entre julho e setembro de 2009. Os dados foram coletados a partir de observação não participante e análise dos prontuários. O formulário utilizado registrou dados sobre a caracterização dos profissionais, técnica de preparo e administração dos medicamentos. Constatouse que $69 \%$ dos fármacos utilizados estavam na forma farmacêutica sólida, submetidos a processos de derivação. Dos medicamentos administrados, 11 (42\%) foram preparados sozinhos, já os demais, 15 (58\%), foram associados a outro medicamento no momento da administração. Destes, 6 preparações continham associações que poderiam ocasionar interação após preparo e administração concomitantes. Concluiu-se que havia desigualdades entre o conhecimento da prática recomendada pela literatura específica e a prática clínica, comprometendo a segurança do paciente.

Descritores: Intubação Gastrointestinal; Interações de Medicamentos; Equipe de Enfermagem; Cuidados de Enfermagem.

\section{ABSTRACT}

The objective of study was to evaluate the performance of nursing aides and technicians in the preparation and administration of drugs delivered via gastrointestinal intubation. This prospective, observational study was performed at a university hospital between July and September of 2009. Data collection was performed by non-participant observation and patient record analysis. A form was used to register the characterization of the professionals and the technique used to prepare and administer drugs. It was found that $69 \%$ of the drugs used were solid, submitted to derivation. Of all the drugs administered, 11 (42\%) were prepared alone, while the other 15 (58\%) were associated to another drug at the time of administration. Six preparations contained ingredients that could cause harmful interactions when simultaneously prepared and administered. It is concluded that there were differences between the practices recommended by the specific literature and actual clinical practices, thus compromising patient safety.

Descriptors: Intubation, Gastrointestinal; Drug Interactions; Nursing, Team; Nursing Care.

\section{RESUMEN}

Se objetivó evaluar el desempeño de técnicos y auxiliares de enfermería durante la preparación y administración de fármacos vía intubación gastrointestinal. Estudio prospectivo, observacional, realizado en hospital universitario ente julio y setiembre de 2009. Datos recolectados mediante observación no participante y análisis de historias clínicas. El formulario utilizado registró datos sobre caracterización de los profesionales, técnicas de preparación y administración de los medicamentos. Se constató que $69 \%$ de los fármacos utilizados eran de forma farmacéutica sólida, sometidos a procesos de derivación. De los medicamentos administrados, 11 (42\%) fueron administrados como monodroga, mientras los otros 15 (58\%) fueron asociados a otro medicamento al momento de la administración. De éstas, seis preparaciones contenían asociaciones que podrían ocasionar interacción luego de la preparación y administración concomitante. Se concluye en que existía desigualdad entre el conocimiento de la práctica recomendada por la literatura específica y la práctica clínica, comprometiéndose la seguridad del paciente.

Descriptores: Intubación Gastrointestinal; Interacciones de Drogas; Grupo de Enfermería; Atención de Enfermería.

\footnotetext{
${ }^{1}$ Enfermeira. Fortaleza, CE, Brasil. E-mail: dayananinha enf@hotmail.com.

${ }^{2}$ Enfermeira, Doutora em Enfermagem. Professor Adjunto da Universidade Federal do Ceará (UFC). Fortaleza, CE, Brasil. E-mail: joselany@ufc.br.

${ }^{3}$ Enfermeira, Mestre em Enfermagem. Fortaleza, CE, Brasil. E-mail: jennaracandido@yahoo.com.br.

${ }^{4}$ Enfermeira. Fortaleza, CE, Brasil. E-mail: bertiane@gmail.com.

${ }^{5}$ Enfermeira, Doutora em Enfermagem. Professor Adjunto da UFC. Fortaleza, CE, Brasil. E-mail: lianinhamara@yahoo.com.br.

${ }^{6}$ Enfermeira. Fortaleza, CE, Brasil. E-mail: mairadi@bol.com.br.
} 


\section{INTRODUÇÃO}

O crescente desenvolvimento farmacológico acarretou inúmeras possibilidades terapêuticas para a prática clínica dos profissionais da saúde. A poliquimioterapia, como estratégia de tratamento, contribui para ampliar os efeitos benéficos da terapia proposta, contudo possibilita a interferência mútua de ações farmacológicas, podendo resultar em alterações dos efeitos desejados ${ }^{(1)}$.

Há evidências da ocorrência de incorreções durante a medicação, decorrentes de falhas individuais, tal como a não observação das especificações referentes a cada medicação, e sistêmicas, como problemas estruturais, e no treinamento de pessoal(2). Estas, potencialmente, danosas para os pacientes que estão sob os cuidados da equipe de enfermagem.

Neste contexto, merece destaque as inadequações que ocorrem durante a administração de medicamentos por intubação gastrointestinal. Embora esta via seja considerada segura e com menor potencial para desenvolvimento de complicações, podem surgir incorreções se não houver planejamento adequado ${ }^{(3-4)}$.

Рara administração de medicação por essa via, devese priorizar o uso de fórmula farmacêutica líquida, no entanto, esta nem sempre se encontra disponível para uso nos serviços de saúde, conduzindo a equipe a optar pela derivação de formas sólidas ${ }^{(5)}$. Esta técnica possui restrições importantes, uma vez que determinados medicamentos não podem ser triturados ou ter seu conteúdo extraído da cápsula por possuírem formulações farmacêuticas especiais, resultando em omissão terapêutica e perda da sonda devido a obstrução(6).

No Brasil, a equipe de enfermagem é responsável pelo preparo, pela administração e pelo monitoramento do paciente em relação aos efeitos do medicamento ${ }^{(7)}$. Ressalta-se que embora estas atividades sejam, frequentemente, delegadas aos técnicos e auxiliares de enfermagem, é responsabilidade do enfermeiro os eventos resultantes deste processo de trabalho. Este responderá pela omissão cometida em suas atividades profissionais, independente de ter sido praticada individualmente ou em equipe ${ }^{(8)}$.

Diante de tamanha responsabilidade, faz-se necessário o desenvolvimento de estudos que avaliem a técnica executada pela equipe durante 0 desenvolvimento das atividades assistenciais, buscando por identificar as lacunas no conhecimento, bem como desequilíbrios, para propor soluções que potencializem o desempenho da equipe de técnicos e auxiliares no que se refere aos processos de preparo e administração de fármacos a pacientes em uso de intubação gastrointestinal.

Diante do exposto, procedeu-se às seguintes proposições: como está sendo realizada a administração de medicamentos via intubação gastrointestinal pela equipe de enfermagem? Existe diferença entre o desempenho realizado e o desempenho esperado? Tendo em vista a variedade de atividades executadas pela equipe de enfermagem, o presente estudo teve como objetivo avaliar o desempenho dos técnicos e auxiliares de enfermagem, durante o preparo e a administração de fármacos por intubação gastrointestinal, de em situação real de assistência.

Acredita-se que o estudo poderá contribuir para melhoria da assistência de enfermagem, oferecendo resultados que auxiliem na adoção de melhores práticas entre técnicos e auxiliares de enfermagem, reduzindo a ocorrência de iatrogênicas, decorrentes de imperícia e imprudência, concorrendo para a segurança dos pacientes nos serviços de saúde.

\section{MATERIAL E MÉTODO}

Estudo descritivo, prospectivo, observacional, que investigou o preparo e a administração de medicamentos realizados por trabalhadores de enfermagem (técnicos e auxiliares) de um hospital universitário, situado em Fortaleza-CE, de julho a setembro de 2009.

A população do estudo foi representada por 27 técnicos e auxiliares de enfermagem que trabalhavam na unidade no período da coleta de dados. O interesse em investigá-los partiu de observações anteriores, não formais, que apontaram o emprego de técnicas inadequadas durante a administração de fármacos via intubação gastrointestinal. Além desse aspecto, merece destacar que a referida unidade se destina ao cuidado de pessoas em situação crítica, possuindo 40 leitos, ofertando as seguintes especialidades: gastroenterologia, nefrologia, infectologia, neurologia e pneumologia. Nesta unidade é grande a variedade de fármacos administrados diariamente, exigindo o 
desempenho de práticas corretas a fim de garantir a segurança dos pacientes sob os cuidados da equipe de enfermagem.

A amostra da pesquisa foi escolhida por conveniência e teve como critérios de inclusão atuar no procedimento de preparo e administração de medicamento na referida clínica e trabalhar no mínimo duas vezes por semana no período da manhã, onde há maior quantidade de medicamentos a serem administrados. Foram desconsiderados todos os funcionários que estavam de licença ou em período de férias, bem como aqueles cuja função não incluía cuidados diretos aos pacientes. Deste modo, a amostra final do estudo foi composta de 22 técnicos e auxiliares de enfermagem.

Os dados foram coletados por meio de observação não participante e direta das técnicas empregadas para administração de medicamentos, via intubação gastrointestinal, e análise de prontuários dos pacientes. O instrumento utilizado para a coleta de dados foi um formulário constituído de duas partes: caracterização do profissional (idade, sexo, categoria profissional e tempo de exercício profissional) e descrição da técnica de preparo e administração dos medicamentos através da intubação gastrointestinal (checagem da prescrição médica, higienização das mãos, separação do material básico, preparação, diluição e administração do medicamento, cuidados com a sonda e registro do procedimento). Para cada item, existiam duas alternativas: desempenho correto e desempenho incorreto, que deveriam ser assinaladas pela pesquisadora no momento da observação do procedimento.

$O$ instrumento de coleta foi submetido à validação aparente e de conteúdo por docentes e enfermeiros do serviço, com vistas a verificar a pertinência e clareza dos itens. Os dados obtidos foram analisados mediante estatística descritiva, sendo posteriormente representados em quadros e tabelas, e discutidos de acordo com aspectos da literatura pertinente.

O estudo foi aprovado pelo Comitê de Ética em Pesquisa da instituição, sob o protocolo $n^{\circ}$ 062.09.08. Todos os participantes do estudo assinaram o Termo de Consentimento Livre e Esclarecido. Também lhes foram garantidos o direito e a liberdade de não participar da pesquisa ou dela retirarem-se a qualquer momento.

\section{RESULTADOS}

Do total de 26 trabalhadores, a maioria era do sexo feminino 19 (73\%), com idades variando entre 20 e 52 anos, sendo 14 (54\%) auxiliares e 12 (46\%) técnicos de enfermagem. Em relação ao tempo de exercício profissional, 20 (77\%) tinham entre um e 10 anos, com média de oito anos.

No decorrer das observações, 45 fármacos foram administrados, sendo que a forma de apresentação do medicamento mais utilizada foi o comprimido, 31 (69\%); seguido pelo uso de formulações líquidas, 10 (22\%); cápsulas, três (7\%); e uma drágea (2\%). Dos medicamentos administrados, 11 (24\%) foram preparados sozinhos; já os demais, 34 (75\%), foram associados a outro medicamento no momento da administração, conforme especificado na Tabela 1.

Tabela 1: Formas de apresentação do medicamento prescrito e o modo de administração via intubação gastrointestinal. Fortaleza, CE, 2009.

\begin{tabular}{|c|c|c|}
\hline Modo de preparo & Forma de apresentação & $\mathbf{N}$ \\
\hline \multirow{4}{*}{ Sem associação entre drogas } & 1 Comprimido & 7 \\
\hline & 1 Líquido & 2 \\
\hline & 1 Cápsula & 1 \\
\hline & 1 Drágea & 1 \\
\hline \multirow{4}{*}{ Duas drogas associadas } & 2 Comprimidos & 7 \\
\hline & 2 Líquidos & 1 \\
\hline & 1 Comprimido + 1 Líquido & 2 \\
\hline & 1 Comprimido + 1 Cápsula & 1 \\
\hline \multirow{3}{*}{ Três drogas associadas } & 3 Comprimidos & 1 \\
\hline & 2 Comprimidos + 1 Líquido & 2 \\
\hline & 1 Comprimido + 2 Líquidos & 1 \\
\hline
\end{tabular}


Os dados da Tabela 2 a seguir referem-se às em uso de intubação gastrointestinal. categorias farmacológicas mais prescritas aos pacientes

Tabela 2: Categorias farmacológicas mais prescritas aos pacientes em uso de intubação gastrointestinal. Fortaleza, CE, 2009.

\begin{tabular}{|c|c|c|}
\hline Categoria farmacológica & $\mathbf{N}$ & $\%$ \\
\hline Antiepilépticos/Anticonvulsivantes & 9 & 53,0 \\
\hline Eletrólitos & 6 & 35,2 \\
\hline Antianêmicos & 4 & 23,5 \\
\hline Anti-hipertensivos & 3 & 5,8 \\
\hline Corticosteroides & 3 & 5,8 \\
\hline Laxantes & 3 & 5,8 \\
\hline Antiarrítmicos & 3 & 5,8 \\
\hline Relaxantes musculares & 2 & 11,7 \\
\hline Anti-inflamatórios não esteroides & 2 & 11,7 \\
\hline Antidepressivos & 2 & 11,7 \\
\hline Antifúngicos & 2 & 11,7 \\
\hline Outros* & 6 & 35,3 \\
\hline
\end{tabular}

o; Antidiarreico; Vasodilatador coronariano

Dentre as várias categorias farmacológicas disponíveis para uso em ambiente hospitalar, os grupos mais prescritos pelos profissionais médicos foram os antiepilépticos/anticonvulsivantes, eletrólitos e antianêmicos. Vale ressaltar que 20 (74,0\%) fármacos estavam disponíveis apenas na apresentação sólida, sendo triturados para posterior administração via intubação gastrointestinal.

Das categorias farmacológicas citadas acima, apenas quatro continham medicamentos na apresentação líquida: antieméticos, antiflatulentos, laxantes e eletrólitos; já as demais estavam disponíveis na unidade apenas na forma de comprimidos e cápsulas. Enfatiza-se que, dentre estes últimos, seis não poderiam ser submetidos à trituração: ácido fólico, ácido acetil salicílico, captopril, clonazepam, fenitoína e risperidona, devido ao risco de obstrução da sonda, além das alterações na farmacocinética da droga decorrentes da quebra.

A Tabela 3 apresenta a avaliação do desempenho dos trabalhadores de enfermagem na execução do preparo e na administração de drogas a serem administradas por intubação gastrointestinal, em situação real de assistência. 
Tabela 3: Etapas seguidas pelos técnicos e/ou auxiliares de enfermagem durante o preparo e a administração de fármacos via intubação gastrointestinal. Fortaleza, CE, 2009.

\begin{tabular}{|c|c|c|c|}
\hline Ação & Desempenho correto & $\mathbf{N}$ & $\%$ \\
\hline Checa a prescrição médica & Checar a prescrição & 25 & 96,0 \\
\hline \multirow[t]{3}{*}{$\begin{array}{l}\text { Lavagem das mãos antes do } \\
\text { procedimento }\end{array}$} & $\begin{array}{l}\text { Lavar as mãos conforme o preconizado pela CCIH da } \\
\text { instituição }\end{array}$ & 4 & 15,0 \\
\hline & Separar o medicamento prescrito & 25 & 96,0 \\
\hline & Separar duas seringas de $20 \mathrm{ml}$ & - & - \\
\hline \multirow[t]{4}{*}{ Separação do material básico } & Separar as luvas de procedimento & 21 & 81,0 \\
\hline & Separar o copo com água & 23 & 88,0 \\
\hline & Separar a etiqueta de identificação & 25 & 96,0 \\
\hline & Não conversar durante o preparo & 6 & 23,0 \\
\hline \multirow[t]{3}{*}{ Preparo do medicamento } & Identificar os copos com o $n^{\circ}$ do leito & 22 & 85,0 \\
\hline & Não tocar o fármaco ao colocá-lo no copo & 17 & 65,0 \\
\hline & Lavar o pilão antes e depois da diluição & 9 & 35,0 \\
\hline \multirow[t]{4}{*}{ Diluição do medicamento } & Macerar os comprimidos separadamente & 8 & 31,0 \\
\hline & Diluir cada comprimido em um copo específico & 5 & 19,0 \\
\hline & Identificar o cliente pelo leito e nome & 26 & 100,0 \\
\hline & Orientar o cliente acerca do procedimento & 26 & 100,0 \\
\hline \multirow{4}{*}{ Administração do medicamento } & Checar o posicionamento da sonda & - & - \\
\hline & Utilizar luvas de procedimento & 21 & 81,0 \\
\hline & $\begin{array}{c}\text { Lavar a sonda entre a administração de um medicamento e } \\
\text { outro }\end{array}$ & 6 & 23,0 \\
\hline & Utilizar no mínimo $20 \mathrm{ml}$ para lavar a sonda & 20 & 77,0 \\
\hline \multirow[t]{2}{*}{ Cuidados com a sonda } & $\begin{array}{c}\text { Fechar a sonda após o uso com tampa específica ou com } \\
\text { seringa }\end{array}$ & 20 & 77,0 \\
\hline & Colocar o paciente em posição de Fowler ou decúbito lateral & 25 & 96,0 \\
\hline \multirow{2}{*}{ Registro do procedimento } & Checar o fármaco administrado & 26 & 100,0 \\
\hline & Registrar o volume no balanço hídrico quando indicado & 26 & 100,0 \\
\hline
\end{tabular}

Observou-se que alguns cuidados ainda não eram adotados por todos os técnicos e auxiliares de enfermagem. Apenas nove $(35,0 \%)$ higienizaram o triturador antes e após o uso, oito $(31,0 \%)$ maceraram os comprimidos separadamente, cinco $(19,0 \%)$ procederam à diluição em separado de fármacos distintos e seis
(23,0\%) lavaram a sonda a cada novo fármaco administrado.

Os dados do Quadro 1 a seguir referem-se as categorias farmacológicas prescritas com risco de interação administrados por SNG/SNE e efeitos esperados.

Quadro 1: Categorias farmacológicas prescritas com risco de interação administrados por SNG/SNE e efeitos esperados. Fortaleza, CE, 2009.

\begin{tabular}{|c|c|}
\hline Categorias Farmacológicas Associadas & Efeitos esperados \\
\hline Antiepiléptico X Antiepilético & Diminuição do efeito \\
\hline Miorrelaxante X Antiepilético & Sinergismo ou efeito aditivo \\
\hline Anti-hipertensivo X Antiepilético & Alteração na concentração final do anti-hipertensivo \\
\hline Corticoesteroide X Antianêmico & O corticoesteroide pode inibir a ação do antianêmico \\
\hline Antiepilético X Anti-hipertensivo & Sinergismo ou efeito aditivo de queda da pressão \\
\hline Antidiarreico X Antiemético & Antagonismo sobre os efeitos sobre a motilidade \\
& gástrica de anticolinérgicos \\
\hline
\end{tabular}


ocasionar interação após o preparo e administração concomitantes.

\section{DISCUSSÃO}

A elevada utilização das formas farmacêuticas sólidas, principalmente comprimidos, drágeas e cápsulas, em pacientes que estão em uso de intubação gastrointestinal representa um risco à saúde, expondo a pessoa à ocorrência de complicações, tal como a perda do tubo por obstrução e falha terapêutica ${ }^{(9)}$. Existe a necessidade de supervisão criteriosa da técnica executada pela equipe de enfermagem durante o preparo e a administração dos medicamentos. Deste modo, o enfermeiro deve orientar sua equipe quanto ao preparo e administração de fármacos, minimizando a ocorrência de erros e resultados inesperados.

Há discrepância entre a técnica recomendada e aquela executada no cotidiano da assistência. Nem sempre a rotina de preparo das medicações nas instituições de saúde obedece às recomendações fornecidas pela indústria farmacêutica, ocasionando falhas $^{(10)}$. Neste estudo, a maioria dos medicamentos utilizados era contraindicados рага uso via intubação gastrointestinal. Preconiza-se o uso de formulações líquidas, em particular elixires e suspensões, para administrar por meio de tubos de alimentação(11). Contudo, determinados medicamentos estão disponíveis apenas como formas farmacêuticas sólidas, e alguns não podem ser triturados.

No entanto, na ausência de formas farmacêuticas líquidas, ou na impossibilidade de optar por outra via de administração, tal como a parenteral ou a retal, poderia ser utilizada a derivação das formas farmacêuticas sólidas, através da trituração(4). A prática de triturar medicamentos, ainda frequente no contexto hospitalar, pode também ter como consequência a interação entre fármacos e a fórmula da nutrição enteral, o que pode levar à diminuição da biodisponibilidade sérica e diminuição da concentração sérica máxima do fármaco e obstrução de sondas, como complicação dessa interação(12).

Estudo realizado a fim de caracterizar os eventos adversos ocorridos com pacientes adultos durante a internação em unidades de terapia intensiva, semiintensiva e internação, evidenciou predomínio de eventos adversos relacionados à sonda nasogástrica
(57,6\%), sendo a obstrução a principal delas, com frequência de 38,5\% na unidade semi-intensiva, 37,50\% na unidade de internação e de $23,20 \%$ na unidade de terapia intensiva ${ }^{(13)}$.

A obstrução da sonda é classificada como uma complicação mecânica, podendo estar relacionada com a retenção de resíduos da fórmula enteral em seu lúmen, em consequência da alta osmolaridade; da formação de complexos insolúveis fórmula-medicamento; de comprimidos macerados inadequadamente e injetados pela sonda e da precipitação da fórmula em razão da acidez do conteúdo gástrico(1).

Esta realidade representa, portanto, um ponto crítico no desempenho das funções da equipe de enfermagem, uma vez que intensificam as chances de interações farmacológicas indesejadas. 0 uso concomitante de vários medicamentos é reconhecido como fator predisponente para a ocorrência de interações farmacêuticas, bem como de outros problemas que irão afetar não apenas o tratamento, como também a saúde de quem venha a receber tal associação(5). Medicações administradas erroneamente podem provocar danos ao paciente decorrentes de incompatibilidade farmacológica ou reações indesejadas ${ }^{(14)}$.

As interações farmacocinéticas provocam alterações na absorção, distribuição, metabolização ou excreção do medicamento. O efeito terapêutico é alcançado, porém sua magnitude e duração podem variar. Já nas interações farmacodinâmicas, há modificação no efeito bioquímico ou fisiológico da droga; geralmente, ocorrem nos receptores farmacológicos, podendo potencializar o efeito da droga (sinergismo) ou diminuí-lo (antagonismo) ${ }^{(15)}$. Pelo exposto, torna-se importante o conhecimento das interações entre medicamentos e entre medicamentos e alimentos para conduzir com segurança os pacientes sob os cuidados da equipe de enfermagem, principalmente tratando-se de polimedicados, idosos e usuários de dietas especiais ${ }^{(16)}$.

Vários estudos ${ }^{(6,11,17)}$ destacam os principais fármacos em apresentações sólidas administrados via intubação gastrointestinal cuja apresentação farmacêutica não deve ser alterada, as alternativas disponíveis para estes casos, além de recomendações para assegurar a efetividade da terapia proposta e melhoria da prática. Dentre as categorias farmacológicas 
que exigem cuidados especiais durante a sua administração, destacam-se: os anticoagulantes, antihipertensivos, antibióticos, antiepiléticos, antiácidos, analgésicos, laxantes, mucolíticos, corticoides, hemostáticos e opiáceos.

Dentre as recomendações gerais, citam-se principalmente a não trituração dos fármacos com revestimento entérico e aqueles de liberação prolongada, adoção de intervalos de administração de uma hora antes a duas horas após a dieta, ou interrupção da infusão enteral contínua por $30 \mathrm{~min}$. A quantidade de líquido recomendada para diluição dos fármacos após a trituração varia, sugerindo que as práticas adotas nas instituições também podem diferir. A lavagem das sondas deve ser realizada antes e após cada nova infusão utilizando volumes que variam entre 10 a $50 \mathrm{ml}$ de água destilada ${ }^{(1,3,9,18-20)}$.

Durante a administração dos medicamentos, os técnicos e auxiliares de enfermagem não demonstraram cuidado de verificar o posicionamento da sonda antes da realização do procedimento. A literatura tem destacado que o mal posicionamento da sonda, decorrente do deslocamento do tubo para fora do estômago ou intestino, pode desencadear reações adversas, tais como: vômitos, tosse, náusea, diarreia, distensão gástrica, ou evoluir para condições de extrema gravidade, tais como bronco-aspiração, pneumonia e sepse ${ }^{(18,21)}$. Portanto, torna-se relevante a observação do posicionamento correto da sonda antes de cada administração.

Outro cuidado igualmente importante para o sucesso da terapia medicamentosa através de sondas, é a manutenção da permeabilidade através da lavagem com 20 a $30 \mathrm{ml}$ de água destilada, antes e após cada administração. Há uma grande necessidade de se evitar as obstruções decorrentes do manejo inadequado, principalmente quando diferentes tipos de fármacos passam a ser administrados via intubação gástrica de uma única vez ${ }^{(3)}$. Neste estudo, a frequência de lavagem da sonda foi realizada na maioria dos casos com $20 \mathrm{ml}$ de água após cada administração.

No concernente aos cuidados após a administração dos fármacos, as observações apontaram que 20 profissionais (77\%) fecharam a sonda com tampa ou seringa, além de colocarem o paciente em posição de Fowler ou em decúbito lateral após o procedimento 25
(96\%). Em relação ao registro, todos checaram o medicamento administrado na prescrição médica e incluíram o volume final no balanço hídrico, quando necessário. Todos esses cuidados são de responsabilidade da equipe de enfermagem e devem ser realizados a fim de evitar outras complicações advindas de manejo inadequado, como aspiração pulmonar.

Em relação às demais condutas adotadas pela equipe durante o preparo dos medicamentos, salienta-se o uso de etiquetas adesivas para a identificação dos fármacos a serem administrados. Durante o preparo do medicamento, 22 (85,0\%) copos utilizados foram identificados apenas com o número do leito, sem menção ao nome do paciente. Vale ressaltar a importância da identificação adequada, uma vez que a numeração do leito não é um dado suficiente, já que trocas podem ser realizadas com frequência em unidades de internação(22). Outra postura que deve ser difundida entre a equipe diz respeito à consulta constante à prescrição, antes, durante e após cada administração, já que o uso de etiquetas não substitui em hipótese a prescrição médica, sob pena de incorrer em erro.

A segurança microbiológica pode estar diminuída quando a enfermagem não limpa a bancada ao preparar medicação, não usa máscara para preparar as soluções, não realiza a desinfecção de ampolas, entre outros

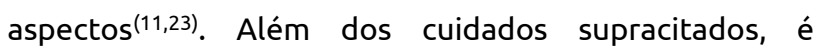
necessária a adoção de outras medidas assépticas durante todo o processo, como a higienização das mãos e o uso de luvas durante a manipulação dos fármacos, após a retirada de sua embalagem original. Durante as observações, apenas 17 (65\%) profissionais não tocaram no comprimido ao colocarem no copo.

Pelo exposto, o conhecimento do enfermeiro e dos demais profissionais de enfermagem, sobre os aspectos ético-legais que envolvem os desvios da qualidade na prestação da assistência, particularmente no processo de medicação, e suas implicações, é de suma importância, tanto como forma de conscientização, quanto para garantir maior segurança na assistência e assegurar os direitos do paciente ${ }^{(24)}$.

Com base nos resultados descritos, percebeu-se que há uma carência no que diz respeito à farmacologia aplicada à prática de enfermagem, podendo comprometer, assim, a terapêutica prescrita. 


\section{CONCLUSÃO}

De acordo com os dados obtidos, verificamos que o desempenho realizado pelos técnicos e auxiliares de enfermagem durante o preparo e a administração de fármacos por intubação gastrointestinal em situação real de assistência diferiu do que é recomendado pela literatura. Observou-se que alguns fatores, como o insuficiente conhecimento específico, contribuem para a perpetuação de técnicas inadequadas de derivação de formas farmacêuticas orais sólidas, possibilitando a ocorrência de interações entre as medicações, perda do tubo, falha na terapêutica medicamentosa.

A crescente utilização da via enteral como meio para administração de fármacos a pacientes graves, bem como em cuidados paliativos, exige a padronização de técnicas para a administração de fármacos, utilizando esta via, reduzindo a possibilidade de erro, garantindo a qualidade do tratamento farmacológico desses pacientes, bem como a segurança.

\section{REFERÊNCIAS}

1. Matsuba CS, De Gutiérrez MG, Whitaker IY. Development and evaluation of standardized protocol to prevent nasoenteral tube obstruction in cardiac patients requiring enteral nutrition with restricted fluid volumes. J Clin Nurs. 2007;16(10):1872-7.

2. Freitas DF, ODA JY. Avaliação dos fatores de risco relacionados às falhas durante a administração de medicamentos. Arq Ciênc Saúde Unipar. 2008;12(3):231-7.

3. Gorzoni ML, Della Torre AP, Sueli L. Drugs and feeding tubes. Rev Assoc Med Bras. 2010;56(1):17-21.

4. Renovato RD, Carvalho PD, Rocha RSA. Investigação da técnica de administração de medicamentos por sondas enterais em hospital geral. Rev Enferm UERJ. 2010; 18(2):173-8.

5. Heydrich J, Heineck I, Bueno D. Observation of preparation and administration of drugs by nursing assistants in patients with enteral feeding tube. Braz J Pharm Sci. 2009;45(1):117-20. 6. Lima G, Negrini MM. Assistência farmacêutica na administração de medicamentos via sonda: escolha da forma farmacêutica adequada. Einstein. 2009;7(1 Pt 1):9-17.

7. Mota MLS, Barbosa IV, Studart RMB, Melo EM, Lima FET, Mariano FA. Avaliação do conhecimento do enfermeiro de unidade de terapia intensiva sobre administração de medicamentos por sonda nasogástrica e nasoenteral. Rev Latino-Am Enfermagem. 2010;18(5):888-94.

8. Conselho Regional de Enfermagem - COREN (BR-SP). Principais legislações para o exercício da enfermagem. São Paulo: COREN 1996.

9. Gómez-Hontanilla M, Ruiz-García MV, Ortega-Delgado J, SalidoZarco A, Gascón-García P. Precauciones en la administración de fármacos por vía enteral. Rev Cient Soc Esp Enferm Neurol. 2010;32:51-54.

10. Corbellini V, Schilling MCL, Frantz SF, Godinho TG, Urbanetto JS. L. Medication-related adverse events: percepception of nursing aides. Rev Bras Enferm. 2011; 64(2):241-7.

11. Silva LD, Lisboa CD. Consequências da interação entre nutrição enteral e fármacos administrados por sondas: uma revisão integrativa. Cogitare Enferm. 2011;16(1):134-40.
Pelo exposto, torna-se necessária a elaboração de um programa de capacitação permanente que venha sanar as deficiências encontradas, em que a equipe de enfermagem tenha espaço para desenvolver competências necessárias ao exercício seguro de suas atribuições nos serviços de saúde.

$\mathrm{Na}$ busca pela excelência em serviço, urge a elaboração de protocolos que auxiliem no processo de preparo e administração de fármacos, com enfoque na escolha da melhor forma farmacêutica para cada via, e da técnica adequada de administração, de modo a evitar problemas decorrentes de incompatibilidades entre as drogas. Também, é preciso supervisão mais assídua pelos enfermeiros do setor sobre as técnicas que estão sendo empregadas para preparar e administrar drogas por sonda pelos trabalhadores de enfermagem, além de mais atenção na execução dos ajustes, com vistas ao não aprazamento concomitante de drogas potencialmente incompatíveis. Dessa forma, pode-se assegurar a eficácia do tratamento, prevenir riscos maiores ao paciente e evitar maiores gastos hospitalares.

12. Malagoli BG, Viel BC, Silva EF, Emery IC, Costa JM, Almeida KCA, Fontes LF, Nascimento MMG. Manual farmacoterapêutico para melhoria das práticas em farmácia hospitalar. Belo Horizonte: UFMG, 2009.

13. Nascimento CCP, Toffoletto MC, Gonçalves LA, Freitas WG, Padilha KG. Indicators of healthcare results: analysis of adverse events during hospital stays. Rev Latino-Am Enfermagem. 2008;16(4):746-51.

14. Silva DO, Grou CR, Miasso Al, Cassiani SHB. Medication preparation and administration: analysis of inquiries and information by the nursing team. Rev Latino-Am Enfermagem. 2007;15(5):1010-7.

15. Rang HP, Dale MM, Ritter JM. Rang\&Dale Farmacologia. $6^{\text {a }}$ ed. Rio de Janeiro: Elsevier; 2008.

16. Magedanz L, Jacoby T, Silva D, Santos L, Martinbiancho J, Zuckermann J. Implementing a program to prevent possible drugfood interactions in adult patients hospitalized in clinical and surgical units of a university hospital. Rev HCPA. 2009;29(1):29-32. 17. Nascimento MMG, Ribeiro AQ. Compilação de base de dados com recomendações para administração de medicamentos via sonda enteral. Brazilian Journal of Hospital Pharmacy and Health Services. 2010;1(1):1-24.

18. Phillips NM, Nay R. A systematic review of nursing administration of medication via enteral tubes in adults. J Clin Nurs. 2008; 17:2257-65.

19. Phillips NM, Endacott R. Medication administration via enteral tubes: a survey of nurses' practices. Journal of Advanced Nursing. 2011;67(12):2586-92.

20. Wohlt PD, Zheng L, Gunderson S, Balzar SA, Johnson BD, Fish JT. Recommendation for the use of medications with continuous enteral nutrition. Am J Health Syst Pharm. 2011;66(16):1458-67. 21. Simons RS, Abdallah LM. Bedside Assessment of Enteral Tube Placement: Aligning Practice with Evidence. The auscultatory method has long been proven unreliable, but old habits die hard. AJN. 2012;112(2):40-46. 
22. Silva AEBC, Cassiani SHB, Miasso Al, Opitz SP. Communication problems: a potential cause for medication error-rates. Acta paul. enferm. 2007;20(3):272-6.

23. Camerini FG, Silva LD. Patient safety: analysing intravenous medication preparation in a sentinel network hospital in brazil. Texto Contexto Enferm. 2011;20(1):41-9.

24. Trevisani Fakih F, Fernandes de Freitas G, Secoli SR.

Medication: ethical and legal aspects for nursing. Rev Bras Enferm. 2009;62(1):132-35.

Artigo recebido em 17/12/2010.

Aprovado para publicação em 25/01/2012.

Artigo publicado em 30/09/2012. 